\title{
Mathematical Models for Continuous Culture Growth Dynamics of Mixed Populations Subsisting on a Heterogeneous Resource Base. II. Predation and Trophic Structure*
}

\author{
Peter E. Smouse \\ Department of Human Genetics, University of Michigan Medical School, \\ Ann Arbor, Michigan 48109
}

Accepted November 7, 1980

\section{INTRODUCTION}

In an earlier paper (Smouse, 1980), I described an extension of the classical model of chemostat dynamics (Monod, 1949), which extension allows one to deal with competion for multiple limiting substrates. The object of the present paper is similarly to extend a simple chemostat model of predation (Tsuchiya et al., 1972) to the case of multiple predators and multiple prey, both underpinned by multiple limiting substrates. I shall also extend the model to multiple trophic layers, and shall indicate the changes necessary to deal with trophic-layer "leap-frogging." In keeping with the strategy of the earlier paper, I shall translate the input parameters of the continuous flow chemostat system into comparable parameters of a modified predation analogue of the sort described by Schoener $(1973,1974)$. This latter type of description, basically an extension of the classic Lotka (1925)-Volterra (1926) predation model, is more familiar to the population biologist. The present treatment may be viewed as a particularization of a more general formulation by Levin et al., (1977), who did not specify the manner in which multiple substrates and multiple prey were to be utilized. It is an alternative to models proposed by Jost et al., (1973), who deal with a different sort of multiple-substrate utilization. There are three questions of primary interest: (1) How do the parameters of the modified predation model relate to those of the chemostat system? (2) What are the parametric conditions for the existence and stability of various possible steady states, and how do these translate into comparable statements about the chemostat

* Supported by NSF-DEB-76-04192. 
system? (3) What may one say about the relative numbers of genotypes (species) maintained in the various trophic layers at equilibrium? I shall attempt to answer each of these questions in turn.

\section{Multiple Substrates and Prey}

A brief recap of the multiple-substrates, multiple-genotypes model of the earlier paper (Smouse, 1980) will facilitate later developments. Consider a series of (prey) genotypes $\left(G_{i}: i=1, \ldots, I\right)$, whose respective masses in the culture vessel are denoted by $\left(M_{i}: i=1, \ldots, I\right)$. These genotypes subsist on and compete for a set of limiting substrates $\left(S_{j}: j=I+1, \ldots, J\right)$, any combination of which is limiting for the growth of any of the $G_{i}$. These substrates are thus alternatives, e.g., different carbon sources, different nitrogen sources, etc.; all other substrates are assumed to be present in considerable excess, and are ignored. The reservoir concentrations of these limiting substrates (determined by the investigator) are denoted by $\left(R_{j}: j=I+1, \ldots, J\right)$, while the culture vessel concentrations (variable) are given by $\left(C_{j}: j=I+1, \ldots, J\right)$. The convention of sequentially numbering genotypes and substrates will avoid confusion below.

The dynamics of the $I$ genotypic components and the $(J-I)$ substrate components are described by Smouse (1980) as

$$
\begin{aligned}
\dot{M}_{i} & =\sum_{j}\left(\mu_{i j}-\gamma_{i j} D\right) M_{i}=\sum_{j} \dot{M}_{i j}=\sum_{j}\left(\frac{V_{i j} C_{j}}{K_{i j} \phi_{i}}-D\right) M_{i} \\
& =\left(\sum_{j} \theta_{i j} C_{j}-D\right) \phi_{i}^{-1} M_{i}, \quad i=1, \ldots, I, \\
\dot{C}_{j} & =\left(R_{j}-C_{j}\right) D-\sum_{i} \mu_{i j} \lambda_{i j} M_{i}, \quad j=I+1, \ldots, J .
\end{aligned}
$$

The term $\mu_{i j}$ is the instantaneous growth rate of $G_{i}$ on $S_{j}$, and $\gamma_{i j}$ is the proportion of the total growth of $G_{i}$ which may be attributed to $S_{j}$; clearly, $\sum_{j} \gamma_{i j}=1$. The parameter $V_{i j}$ is the corresponding maximum achievable growth rate; $K_{i j}$ is the "half-maximum" substrate concentration, i.e., that $C_{j}$ such that $\mu_{i j}=V_{i j} / 2 ; \theta_{i j}=\left(V_{i j}-D\right) / K_{i j}$ is a convenient transform, perhaps best viewed as a standardized growth measure. The parameter $\lambda_{i j}$ measures the number of units of $S_{j}$ required to produce one unit of $G_{i}$, an inverse measure of conversion efficiency. The term $\phi_{i}=\left[1+\sum_{j} K_{i j}^{-1} C_{j}\right]$ changes with the state of the system, but conveniently cancels from much of what follows. The parameter $D$ is the volumetric dilution rate of the chemostat system, and is under cxperimental control. 
In addition to (1), a set of "state" equations may also be described. These take the form

$$
\left[\dot{C}_{j}+\sum_{j} \lambda_{i j} \dot{M}_{i j}\right]=\left[R_{j}-C_{j}-\sum_{i} \Gamma_{i j} M_{i}\right] D \approx 0, \quad j=I+1, \ldots, J,
$$

where $\Gamma_{i j}=\lambda_{i j} \gamma_{i j}$. Equations (2) describe the residual amounts of substrate in the culture vessel, measured either directly or in terms of biomass equivalents. If initially the $M_{i} \sim 0$ and the $C_{j} \sim R_{j}$, Eqs. (2) begin and remain nearly zero. This is the usual case for chemostat cultures (Waldon, 1975). Substitution of (2) into (1) yields the approximate Lotka (1925)-Volterra (1926) form

$$
\begin{gathered}
\dot{M}_{i}=\left[\alpha_{i}-\sum_{i^{\prime}} \beta_{i i^{\prime}} M_{i^{\prime}}\right] \phi_{i}^{-1} M_{i}, \\
\alpha_{i}=\left(\sum_{j} \theta_{i j} R_{j}-D\right), \quad\left(\sum_{j} \theta_{i j} \Gamma_{i^{\prime} j}\right)=\beta_{i i^{\prime}}, \\
i, i^{\prime}=1, \ldots, I .
\end{gathered}
$$

The approximation arises from the fact that the $\phi_{i}$ are not constants. The conditions for the existence of the various steady states may be described in terms of the usual Lotka-Volterra conditions on the $\alpha$ - and $\beta$-parameters. Stability conditions also involve the $\phi_{i}$-measures (cf. Strobeck, 1973).

A detailed exposition of this model may be found in the earlier paper (Smouse, 1980), but one feature is particularly worth mentioning here. The matrix $B$ of $\beta$-coefficients is crucial to these existence and stability arguments. This matrix B may be partitioned into $(J-I)$ separate matrices $\mathbf{B}_{j}$, one for each substrate; the matrix $\mathbf{B}_{j}$ is defined by

$$
\mathbf{B}_{j}=\operatorname{diag}\left\{\theta_{i j}\right\}\left[\begin{array}{ccc}
1 & \cdots & 1 \\
\vdots & & \vdots \\
1 & \cdots & 1
\end{array}\right] \operatorname{diag}\left\{\Gamma_{i j}\right\},
$$

and is of unit rank only. If we adopt the conventions that any two substrates perceived identically by the genotypes are the same and that any two genotypes having identical perceptions of the substrates are the same, then the matrix $\mathrm{B}$ is of rank $H=\min (I, J-I)$, although of order $I$. A minimal condition for the existence of an $I$-genotype mix is that the $\mathbf{B}$ matrix be of full rank (Strobeck, 1973). Thus, we must have $I \leqslant H$ for all $I$ genotypes to persist; this is a necessary, but not sufficient condition. One cannot maintain more genotypes than substrates, given that the population is limited by these substrates. 


\section{Simple Predation}

One predator, one prey, one substrate. It is convenient to begin with the simple model of Tsuchiya et al., (1972). Consider a single predator $\left(G_{1}\right)$, whose limiting substrate is the single prey $\left(G_{2}\right)$. This latter is assumed to be dependent on a single growth limiting substrate $\left(S_{3}\right)$. A schematic is provided in Fig. 1a. In the present notation, the dynamic and state equations are

$$
\begin{gathered}
\dot{M}_{1}=\left(\mu_{12}-D\right) M_{1}=\left[\frac{V_{12} M_{2}}{K_{12} \phi_{1}}-D\right] M_{1}=\left(\theta_{12} M_{2}-D\right) \phi_{1}^{-1} M_{1}, \\
\dot{M}_{2}=\left(\mu_{23}-D\right) M_{2}-\mu_{12} \lambda_{12} M_{1}=\left[\frac{V_{23} C_{3}}{K_{23} \phi_{2}}-\frac{\lambda_{12} V_{12} M_{1}}{K_{12} \phi_{1}}-D\right] M_{2}, \\
\dot{C}_{3}=\left(R_{3}-C_{3}\right) D-\mu_{23} \lambda_{23} M_{2}, \\
{\left[\dot{C}_{3}+\lambda_{23}\left(\dot{M}_{2}+\lambda_{12} \dot{M}_{1}\right)\right]=\left[R_{3}-C_{3}-\lambda_{23}\left(M_{2}+\lambda_{12} M_{1}\right)\right] D \approx 0,}
\end{gathered}
$$

where $\phi_{1}=\left[1+K_{12}^{-1} M_{2}\right], \theta_{12}=\left(V_{12}-D\right) / K_{12}$ and $\phi_{2}=\left[1+K_{23}^{-1} C_{3}\right]$. The definitions of all the other terms are obvious. Substitution of $C_{3}$ from the state equation into the $\dot{M}_{2}$ equation (5) yields a modified predation model
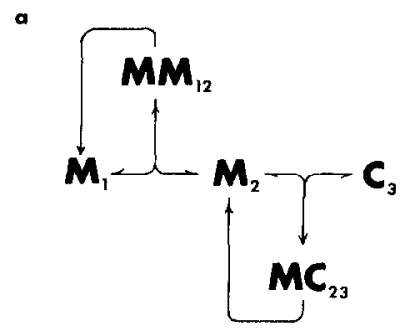

b

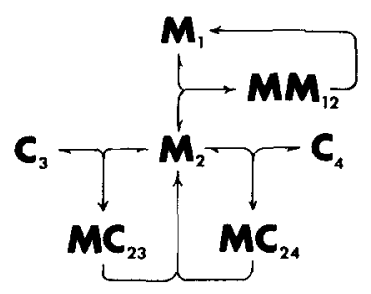

FIG. 1. Simple predation schematics: (a) $M_{1}=$ mass of the predator, $M_{2}=$ mass of the prey, $C_{3}=$ concentration of the single limiting substrate; (b) $M_{1}$ and $M_{2}$ as in (a), $C_{3}$ and $C_{4}=$ concentration of alternate limiting substrates. 
(cf. Canale, 1969, 1970; Schoener, 1973, 1974). In the present notation, this model takes the form

$$
\begin{aligned}
& \dot{M}_{1}=\left[-\alpha_{1}+\beta_{12} M_{2}\right] M_{1}, \\
& \dot{M}_{2}=\left[\alpha_{2}-\beta_{21} M_{1}-\beta_{22} M_{2}\right] M_{2},
\end{aligned}
$$

with the $\alpha$ - and $\beta$-coefficients given by

$$
\begin{gathered}
\alpha_{1}=D, \quad \alpha_{2}=\frac{V_{23} R_{3}}{K_{23} \phi_{2}}-D, \\
\beta_{12}=\frac{V_{12}}{K_{12} \phi_{1}}, \quad \beta_{22}=\frac{V_{23} \lambda_{23}}{K_{23} \phi_{2}}, \quad \beta_{21}=\lambda_{12}\left(\beta_{12}+\beta_{22}\right) .
\end{gathered}
$$

Two features are of immediate interest. First, the self-damping term for the prey $\left(-\beta_{22} M_{2}^{2}\right)$ is not present in the classical predation model (Gause, 1934), but is intrinsic in this situation, since the prey is itself utilizing an exhaustable resource $\left(S_{3}\right)$. Second, the model is not really a quadratic differential system, since the $\alpha$ - and $\beta$-coefficients depend on $C_{3}$ and $M_{2}$, which are themselves variable. It is nevertheless convenient to write the model in this form.

One predator, one prey, multiple substrates. The first generalization of the model is to allow for multiple substrates $\left(S_{j}: j=3, \ldots, J\right)$ underpinning the prey organism. A schematic for two substrates is presented in Figure lb. In the more general case, the system (5) is replaced by the analogous set

$$
\begin{gathered}
\dot{M}_{1}=\left(\mu_{12}-D\right) M_{1}=\left[\frac{V_{12} M_{2}}{K_{12} \phi_{1}}-D\right] M_{1}=\left(\theta_{12} M_{2}-D\right) \phi_{1}^{-1} M_{1}, \\
\dot{M}_{2}=\frac{V}{j}\left[\frac{V_{2 j} C_{j}}{K_{2 j} \phi_{2}}-\gamma_{2 j}\left(D+\frac{\lambda_{12} V_{12}}{K_{12} \phi_{1}} M_{1}\right)\right] M_{2}=\sum_{j} \dot{M}_{2 j}, \\
\dot{C}_{j}=\left(R_{j}-C_{j}\right) D-\mu_{2 j} \lambda_{2 j} M_{2}, \quad j=3, \ldots, J, \\
\left.\dot{C}_{j}+\lambda_{2 j}\left(\dot{M}_{2 j}+\gamma_{2 j} \lambda_{12} \dot{M}_{1}\right)\right] \\
=\left[R_{j}-C_{j}-\Gamma_{2 j}\left(M_{2}+\lambda_{12} M_{1}\right)\right] D \approx 0, \quad j=3, \ldots, J,
\end{gathered}
$$

where $\phi_{1}=\left[1+K_{12}^{-1} M_{2}\right]$ and $\phi_{2}=\left[1+\sum_{j} K_{2 j}^{-1} C_{j}\right]$. We are led again to the predator-prey model (6), but now with $\alpha$ - and $\beta$-coefficients defined by

$$
\begin{gathered}
\alpha_{1}=D, \quad \alpha_{2}=\sum_{j} \frac{V_{2 j} R_{j}}{K_{2 j} \phi_{2}}-D, \\
\beta_{12}=\frac{V_{12}}{K_{12} \phi_{1}}, \quad \beta_{22}=\sum_{j} \frac{V_{2 j} \Gamma_{2 j}}{K_{2 j} \phi_{2}}, \quad \beta_{21}=\lambda_{12}\left(\beta_{12}+\beta_{22}\right) .
\end{gathered}
$$


The essence of the model is contained in the criteria for existence and stability of the various steady-state solutions to (6). Local stability may be evaluated in the usual Liapunov fashion (Canale, 1969, 1970). The criteria for existence and stability of the three potential steady states are listed below. [The stability conditions are obtained as illustrated in the Appendix.]

Case I.

$$
\hat{M}_{1}=0=\hat{M}_{2}, \quad \hat{C}_{j}=R_{j}, \quad j=3, \ldots, J .
$$

Exists if: Always.

Stable if: $\alpha_{2}=\left(\sum_{j} \theta_{2 j} R_{j}-D\right)<0$ (Washout conditions).

Case II.

$$
\hat{M}_{1}=0, \quad \hat{M}_{2}=\frac{\alpha_{2}}{\beta_{22}}=\frac{\left(\sum_{j} \theta_{2 j} R_{j}-D\right)}{\sum_{j} \theta_{2 j} \Gamma_{2 j}}, \quad \sum_{j} \theta_{2 j} \hat{C}_{j}=D .
$$

Exists if: $\alpha_{2}=\left(\sum_{j} \theta_{2 j} R_{J}-D\right)>0$ (Growth conditions).

Stable if: $0<\frac{\left(\sum_{j} \theta_{2 j} R_{j}-D\right)}{\sum_{j} \theta_{2 j} \Gamma_{2 j}}=\frac{\alpha_{2}}{\beta_{22}}<D \theta_{12}^{-1}=\frac{\alpha_{1}}{\beta_{12}}$.

Case III.

$$
\begin{aligned}
& \hat{M}_{1}=\frac{\alpha_{2} \beta_{12}-\alpha_{1} \beta_{22}}{\beta_{12} \beta_{21}}=\lambda_{12}^{-1}\left[\sum_{j} \frac{R_{j}-\hat{C}_{j}}{\lambda_{2 j}}-D \theta_{12}^{-1}\right], \\
& \hat{M}_{2}=\frac{\alpha_{1}}{\beta_{12}}=\frac{D K_{12}}{V_{12}-D}=D \theta_{12}^{-1} \quad \sum_{j} \frac{V_{2 j} C_{j}}{K_{2 j} \phi_{2}}=\theta_{12} \sum_{j} \frac{R_{j}-\hat{C}_{j}}{\lambda_{2 j}} . \\
& \text { Exists if: } \frac{\alpha_{2}}{\beta_{22}}=\frac{\left(\sum_{j} \theta_{2 j} R_{j}-D\right)}{\sum_{j} \theta_{2 j} \Gamma_{2 j}}>0<D \theta_{12}^{-1}=\frac{\alpha_{1}}{\beta_{12}} . \\
& \text { Stable if: } \frac{\alpha_{2}}{\beta_{22}}=\frac{\left(\sum_{j} \theta_{2 j} R_{j}-D\right)}{\sum_{j} \theta_{2 j} \Gamma_{2 j}}>D \theta_{12}{ }^{1}=\frac{\alpha_{1}}{\beta_{12}}>0 .
\end{aligned}
$$

With a single substrate, the subscript $(j)$ takes only the value " 3 ," and one may drop the summations over $(j)$ in the above.

The three solution sets are mutually exclusive as regards stability. Under washout conditions, the null solution (Case I) is stable; under growth conditions, either Case II or Case III is stable. Unless the prey are more dense in the presence of the predator than in its absence, a highly unlikely situation, Case III is stable. Limit cycles are not inherent in this model, due 
to the self-damping term $\left(-\beta_{22} M_{2}^{2}\right)$. The Kolmogorov Theorem (cf. May, 1973) guarantees the existence of either a stable equilibrium or a stable limit cycle under certain parametric conditions, but these conditions are only satisfied by Case III. (See also Canale 1969, 1970.)

One predator, multiple prey, multiple substrates. The next generalization is the multiple prey case. Denote the predator by $\left(G_{1}\right)$, the several prey by $\left(G_{i}: i=2, \ldots, I\right)$, and the substrates by $\left(S_{j}: j=I+1, \ldots, J\right)$. A schematic for two prey and two substrates is presented as Figure 2. In keeping with the analogous competition models (Smouse, 1980)), it is convenient to view these prey as alternate substrates for the predator. The system is described by

$$
\begin{aligned}
& \dot{M}_{1}=\sum_{i}\left(\mu_{1 i}-\gamma_{1 i} D\right)=\sum_{i}\left[\frac{V_{1 i} M_{i}}{K_{1 i} \phi_{1}}-\gamma_{1 i} D\right] M_{1}=\sum_{i} \dot{M}_{1 i} \\
& \dot{M}_{i}=\sum_{j}\left[\frac{V_{i j} C_{j}}{K_{i j} \phi_{i}}-\gamma_{i j}\left(D+\frac{\lambda_{1 i} V_{1 i}}{K_{1 i} \phi_{1}} M_{1}\right)\right] M_{i}=\sum_{j} \dot{M}_{i j}, \quad i=2, \ldots, I \\
& \dot{C}_{j}=\left(R_{j}-C_{j}\right) D-\sum_{i} \mu_{i j} \lambda_{i j} M_{i}, \quad j=I+1, \ldots, J \\
& {\left[\dot{C}_{j}+\sum_{i} \lambda_{i j}\left(\dot{M}_{i j}+\gamma_{i j} \lambda_{1 i} \dot{M}_{1 i}\right)\right]} \\
& \quad=\left[R_{j}-C_{j}-\sum_{i} \Gamma_{i j}\left(M_{i}+\Gamma_{1 i} M_{1}\right)\right] D \approx 0, \quad j=I+1, \ldots, J
\end{aligned}
$$

Here $\phi_{1}=\left[1+\sum_{i} K_{1 i}^{-1} M_{i}\right]$ and $\phi_{i}=\left[1+\sum_{j} K_{i j}^{-1} C_{j}\right]$. All the other parameters have obvious definitions. It should come as no surprise that we are led directly to the modified predation model

$$
\begin{gathered}
\dot{M}_{1}=\left[-\alpha_{1}+\sum_{i} \beta_{1 i} M_{i}\right] M_{1}, \\
\dot{M}_{i}=\left[\alpha_{i}-\beta_{i 1} M_{1}-\sum_{i^{\prime}} \beta_{i i^{\prime}} M_{i^{\prime}}\right] M_{i}, \quad i, i^{\prime}=2, \ldots, I,
\end{gathered}
$$

with the $\alpha$ - and $\beta$-coefficients defined by

$$
\begin{gathered}
\alpha_{1}=D, \\
\alpha_{i}=\left(\sum_{j} \frac{V_{i j} R_{j}}{K_{i j} \phi_{j}}-D\right), \quad \beta_{1 i}=\frac{V_{1 i}}{K_{1 i} \phi_{1}}, \quad i=2, \ldots, I, \\
\beta_{i i^{\prime}}=\sum_{j} \frac{V_{i j} \Gamma_{i^{\prime} j}}{K_{i j} \phi_{i}}, \quad \beta_{i 1}=\lambda_{1 i} \beta_{1 i}+\sum_{i^{\prime}} \Gamma_{1 i}, \beta_{i i^{\prime}}, \quad i, i^{\prime}=2, \ldots, I .
\end{gathered}
$$




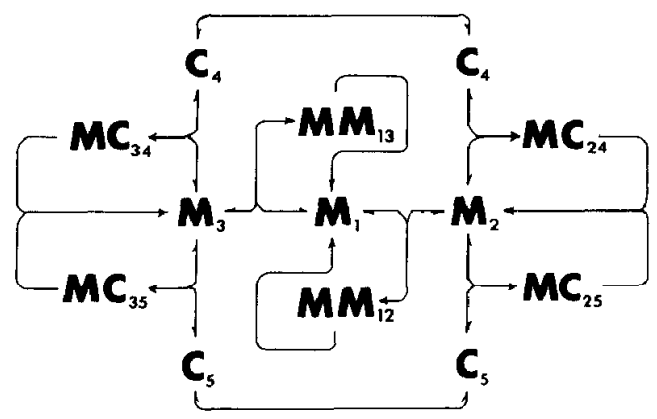

FIG. 2. One predator, two prey, two substrate model: $M_{1}=$ mass of the predator, $M_{2}$ and $M_{3}=$ masses of the two prey, $C_{4}$ and $C_{5}=$ concentrations of the alternate limiting substrates.

There are $\left(2^{I}-1\right)$ solution sets for $(12)$; we cannot have persistence of only the predator. An exhaustive treatment of all of these possibilities is beyond the scope of this paper. [The reader interested in the general features of local and global stability analysis is referred to Gilpin (1974) and Goh (1977).] The seven solution sets of the one predator, two prey model should convey the general flavor of the situation, however, and are therefore presented below.

Case I.

$$
\hat{M}_{i}=0, \quad i=1, \ldots, 3, \quad \hat{C}_{j}=R_{j}, \quad j=4, \ldots, J .
$$

Exists if: Always.

Stable if: $\left(\sum_{j} \theta_{2 j} R_{j}-D\right)=\alpha_{2}<0>\alpha_{3}=\left(\sum_{j} \theta_{3 j} R_{j}-D\right)$ (Washout).

Case II.

$\hat{M}_{1}=0, \quad \hat{M}_{2}=\frac{\alpha_{2}}{\beta_{22}}=\frac{\sum_{j} \theta_{2 j} R_{j}-D}{\sum_{j} \theta_{2 j} \Gamma_{2 j}}, \quad \hat{M}_{3}=0, \quad \sum_{j} \theta_{2 j} \hat{C}_{j}=D$.

Exists if: $\left(\sum_{j} \theta_{2 j} R_{j}-D\right)=\alpha_{2}>0$ (Growth).

Stable if: $\frac{\sum_{j} \theta_{3 j} R_{j}-D}{\sum_{j} \theta_{3 j} \Gamma_{2 j}}=\frac{\alpha_{3}}{\beta_{32}}<\frac{\alpha_{2}}{\beta_{22}}=\frac{\sum_{j} \theta_{2 j} R_{j}-D}{\sum_{j} \theta_{2 j} \Gamma_{2 j}}<D \theta_{12}^{-1}=\frac{\alpha_{1}}{\beta_{12}}$.

Case III.

$$
\hat{M}_{1}=0, \quad \hat{M}_{2}=0, \quad \hat{M}_{3}=\frac{\alpha_{3}}{\beta_{33}}=\frac{\sum_{j} \theta_{3 j} R_{j}-D}{\sum_{j} \theta_{3 j} \Gamma_{3 j}}, \quad \sum_{j} \theta_{3 j} \hat{C}_{j}=D .
$$


Exists if: $\left(\sum_{j} \theta_{3 j} R_{j}-D\right)=\alpha_{3}>0$ (Growth).

Stable if: $\frac{\sum_{j} \theta_{2 j} R_{j}-D}{\sum_{j} \theta_{2 j} \Gamma_{3 j}}=\frac{\alpha_{2}}{\beta_{23}}<\frac{\alpha_{3}}{\beta_{33}}=\frac{\sum_{j} \theta_{3 j} R_{j}-D}{\sum_{j} \theta_{3 j} \Gamma_{3 j}}<D \theta_{13}^{-1}=\frac{\alpha_{1}}{\beta_{13}}$.

Case IV.

$$
\begin{aligned}
\hat{M}_{1}=0, \quad \hat{M}_{2}= & \frac{\alpha_{2} \beta_{33}-\alpha_{3} \beta_{23}}{\beta_{22} \beta_{33}-\beta_{23} \beta_{32}}>0<\frac{\alpha_{3} \beta_{22}-\alpha_{2} \beta_{32}}{\beta_{22} \beta_{33}-\beta_{23} \beta_{32}}=\hat{M}_{3}, \\
& \sum_{j} \theta_{2 j} \hat{C}_{j}=D=\sum_{j} \theta_{3 j} \hat{C}_{j} .
\end{aligned}
$$

Exists if: $\left(\sum_{j} \theta_{2 j} R_{j}-D\right)=\alpha_{2}>0<\alpha_{3}=\left(\sum_{j} \theta_{3 j} R_{j}-D\right)$.

Stable if: $\frac{\alpha_{2}}{\beta_{23}}>\frac{\alpha_{3}}{\beta_{33}}>0<\frac{\alpha_{2}}{\beta_{22}}<\frac{\alpha_{3}}{\beta_{32}}, \quad \beta_{22} \beta_{33}>\beta_{23} \beta_{32}$,

$$
\hat{M}_{2} \theta_{13}^{-1}+\hat{M}_{3} \theta_{12}^{-1}<D \theta_{12}^{-1} \theta_{13}^{-1} \text {. }
$$

Case V.

$$
\begin{gathered}
\hat{M}_{1}=\frac{\alpha_{2} \beta_{12}-\alpha_{1} \beta_{22}}{\beta_{12} \beta_{21}}=\frac{1}{\lambda_{12}}\left[\sum_{j} \frac{R_{j}-\hat{C}_{j}}{\lambda_{2 j}}-D \theta_{12}^{-1}\right], \quad \hat{M}_{3}=0, \\
\hat{M}_{2}=\frac{\alpha_{1}}{\beta_{12}}-D \theta_{12}^{-1}, \quad \sum_{j} \frac{V_{2 j} \hat{C}_{j}}{K_{2 j}+\hat{C}_{j}}=\theta_{12} \sum_{j} \frac{R_{j}-\hat{C}_{j}}{\lambda_{2 j}} .
\end{gathered}
$$

Exists if: $\left(\sum_{j} \theta_{2 j} R_{j}-D\right)=\alpha_{2}>0<D \theta_{12}^{-1}=\frac{\alpha_{1}}{\beta_{12}}$.

Stable if: $\frac{\alpha_{2} \beta_{31}-\alpha_{3} \beta_{21}}{\beta_{22} \beta_{31}-\beta_{21} \beta_{32}}>\frac{\alpha_{1}}{\beta_{12}}=D \theta_{11}^{-1}<\frac{\sum_{j} \theta_{2 j} R_{j}-D}{\sum_{j} \theta_{2 j} \Gamma_{2 j}}=\frac{\alpha_{2}}{\beta_{22}}$.

[Note that $\hat{\phi}_{1}=\left(1+K_{12}^{-1} \hat{M}_{2}\right)=V_{12} /\left(V_{12}-D\right)$.]

Case VI.

$$
\begin{gathered}
\hat{M}_{1}=\frac{\alpha_{3} \beta_{13}-\alpha_{1} \beta_{33}}{\beta_{13} \beta_{31}}=\frac{1}{\lambda_{13}}\left[\sum_{j} \frac{R_{j}-\hat{C}_{j}}{\lambda_{3 j}}-D \theta_{13}^{-1}\right], \quad \hat{M}_{2}=0, \\
\hat{M}_{3}=\frac{\alpha_{1}}{\beta_{13}}=D \theta_{13}^{-1}, \quad \sum_{j} \frac{V_{3 j} \hat{C}_{j}}{K_{3 j}+\hat{C}_{j}}=\theta_{13} \sum_{j} \frac{R_{j}-\hat{C}_{j}}{\lambda_{3 j}} . \\
\text { Exists if: }\left(\sum_{j} \theta_{3 j} R_{j}-D\right)=\alpha_{3}>0<D \theta_{13}^{-1}=\frac{\alpha_{1}}{\beta_{13}} .
\end{gathered}
$$


Stable if: $\frac{\alpha_{2} \beta_{31}-\alpha_{3} \beta_{21}}{\beta_{23} \beta_{31}-\beta_{21} \beta_{33}}>\frac{\alpha_{1}}{\beta_{13}}=D \theta_{13}^{-1}<\frac{\sum_{j} \theta_{3 j} R_{j}-D}{\sum_{j} \theta_{3 j} \Gamma_{3 j}}=\frac{\alpha_{3}}{\beta_{33}}$.

[Note that $\hat{\phi}_{1}=\left(1+K_{13}^{-1} \hat{M}_{3}\right)=V_{13} /\left(V_{13}-D\right)$.]

Case VII.

$$
\begin{aligned}
\hat{M}_{1}= & {\left[-\alpha_{1}\left(\beta_{22} \beta_{33}-\beta_{23} \beta_{32}\right)+\beta_{12}\left(\alpha_{2} \beta_{33}-\alpha_{3} \beta_{23}\right)\right.} \\
& \left.+\beta_{13}\left(\alpha_{3} \beta_{22}-\alpha_{2} \beta_{32}\right)\right] \operatorname{det} \mathbf{B}^{-1}, \\
\hat{M}_{2}= & {\left[\alpha_{1}\left(\beta_{21} \beta_{33}-\beta_{23} \beta_{31}\right)-\beta_{13}\left(\alpha_{3} \beta_{21}-\alpha_{2} \beta_{31}\right)\right] \operatorname{det} \mathbf{B}^{-1}, } \\
\hat{M}_{3}= & {\left[\alpha_{1}\left(\beta_{22} \beta_{31}-\beta_{21} \beta_{32}\right)-\beta_{12}\left(\alpha_{2} \beta_{31}-\alpha_{3} \beta_{21}\right)\right] \operatorname{det} \mathbf{B}^{-1}, } \\
\operatorname{det} \mathbf{B}= & {\left[\beta_{12}\left(\beta_{21} \beta_{33}-\beta_{23} \beta_{31}\right)+\beta_{13}\left(\beta_{22} \beta_{31}-\beta_{21} \beta_{32}\right)\right], } \\
\hat{C}_{j}=R_{j}- & \Gamma_{2 j}\left(\hat{M}_{2}+\Gamma_{12} \hat{M}_{1}\right)-\Gamma_{3 j}\left(\hat{M}_{3}+\Gamma_{13} \hat{M}_{1}\right), \quad j=4, \ldots, J .
\end{aligned}
$$

Exists if: $\hat{M}_{1}, \hat{M}_{2}, \hat{M}_{3}>0$.

Stable if: $\hat{M}_{2} \hat{M}_{3}\left(\beta_{22} \beta_{33}-\beta_{23} \beta_{32}\right)+\hat{M}_{1} \hat{M}_{2} \beta_{12} \beta_{21}+\hat{M}_{1} \hat{M}_{3} \beta_{13} \beta_{31}$

$$
>0<\operatorname{det} \mathrm{B} \cdot \hat{M}_{1} \hat{M}_{2} \hat{M}_{3} \text {. }
$$

It can be shown that if Case VII exists and is stable, Cases IV, V and VI are unstable. If Case VII exists, but is unstable, then Cases IV, V and VI are stable. Cases II and V cannot both be stable, nor can Cases III and VI. Moreover, Case VI cannot be jointly stable with Cases II or III. Case I is stable only under generalized washout conditions.

It is instructive to examine these various solution sets when only a single substrate $\left(S_{4}\right)$ is present. Cases I-III ate possible, as are Cases V and VI. Case IV cannot exist, however, because

$$
\left(\beta_{22} \beta_{33}-\beta_{23} \beta_{32}\right)=\frac{V_{24} \Gamma_{24}}{K_{24} \phi_{2}} \cdot \frac{V_{34} \Gamma_{34}}{K_{34} \phi_{3}}-\frac{V_{24} \Gamma_{34}}{K_{24} \phi_{2}} \cdot \frac{V_{34} \Gamma_{24}}{K_{34} \phi_{3}}=0 .
$$

Note, however, that even if (13) is zero, the full $(3 \times 3)$ matrix $B$ is of rank 3 , because it may be partitioned as two separate matrices $\mathbf{B}=\mathbf{B}_{1}+\mathbf{B}_{4}^{*}$. The matrix $\mathbf{B}_{1}$ is given by

$$
\mathbf{B}_{1}=\left[\begin{array}{ccc}
0 & \beta_{12} & \beta_{13} \\
\lambda_{12} \beta_{12} & 0 & 0 \\
\lambda_{13} \beta_{13} & 0 & 0
\end{array}\right],
$$

and is of rank 2, while the matrix $\mathbf{B}_{4}^{*}$ is given by

$$
\mathbf{B}_{4}^{*}=\left[\begin{array}{c:c}
0 & 0 \\
\hdashline \widetilde{\mathbf{B}}_{4} & \mathbf{B}_{4}
\end{array}\right]=\left[\begin{array}{c:cc}
0 & 0 & 0 \\
\hdashline \Gamma_{12} \beta_{22}+\Gamma_{13} \beta_{23} & \beta_{22} & \beta_{23} \\
\Gamma_{12} \beta_{32}+\Gamma_{13} \beta_{33} & \beta_{32} & \beta_{33}
\end{array}\right],
$$


and is of the same rank as $\mathbf{B}_{4}$, i.e., unity. Thus, Case VII may be stable, even though Case IV is not. The presence of a predator may stabilize an otherwise unstable pair of prey.

In the more general case of one predator, multiple prey, multiple substrates, the matrix $\mathbf{B}$ is of rank $(J-I+2)$, since $\mathbf{B}=\mathbf{B}_{1}+\sum_{j} \mathbf{B}_{j}^{*}$, with $\mathbf{B}_{1}$ of rank 2 and each of the $(J-I)$ matrices B* of rank 1. Thus, if $N_{J}=(J-I)$ is the number of substrates, the maximum number of prey is $N_{I}=(I-1) \leqslant$ $N_{J}+1$, I shall generalize this statement in the next section.

Multiple predators, multiple prey, multiple substrates. Now, consider a set of predators $\left(G_{h}: h=1, \ldots, H\right)$, a set of prey $\left(G_{i}: i=H+1, \ldots, I\right)$, and a set of substrates $\left(S_{j}: j=I+1, \ldots, J\right)$. The system is described by

$$
\begin{aligned}
& \dot{M}_{h}=\sum_{i}\left[\frac{V_{h i} M_{i}}{K_{h i} \phi_{h}}-\gamma_{h i} D\right] M_{h}=\sum_{i} \dot{M}_{h i}, \quad h=1, \ldots, H \\
& \dot{M}_{i}=\sum_{j}\left[\frac{V_{i j} C_{j}}{K_{i j} \phi_{i}}-\gamma_{i j}\left(D+\sum_{h} \frac{V_{h i} \lambda_{h i}}{K_{h i} \dot{\phi}_{h}} M_{h}\right)\right] M_{i}=\sum_{j} \dot{M}_{i j}, \quad i=H+1, \ldots, I \\
& \dot{C}_{j}=\left(R_{j}-C_{j}\right) D-\sum_{i} \mu_{i j} \lambda_{i j} M_{i}, \quad j=I+1, \ldots, J \\
& {\left[\dot{C}_{j}+\sum_{i} \lambda_{i j}\left(\dot{M}_{i j}+\gamma_{i j} \sum_{h} \lambda_{h i} \dot{M}_{h i}\right)\right]} \\
& \quad=\left[R_{j}-C_{j}-\sum_{i} \Gamma_{i j}\left(M_{i}+\sum_{h} \Gamma_{h i} M_{h}\right)\right] D \approx 0, \quad j=I+1, \ldots, J .
\end{aligned}
$$

Here $\phi_{h}=\left[1+\sum_{i} K_{h i}^{-1} M_{i}\right]$ and $\phi_{i}=\left[1+\sum_{j} K_{i j}^{-1} C_{j}\right]$. We are led directly to the generalized predator-prey system

$$
\begin{aligned}
& \dot{M}_{h}=\left[-\alpha_{h}+\sum_{i} \beta_{h i} M_{i}\right] M_{h}, \quad h=1, \ldots, H, \\
& \dot{M}_{i}=\left[\alpha_{i}-\sum_{h} \beta_{i h} M_{h}-\sum_{i^{\prime}} \beta_{i i^{\prime}} M_{i^{\prime}}\right] M_{i}, \quad i=H+1, \ldots, I .
\end{aligned}
$$

For the present model, the $\alpha$ - and $\beta$-coefficients take the obvious forms

$$
\begin{gathered}
\alpha_{h}=D, \quad \beta_{h i}=\frac{V_{h i}}{K_{h i} \phi_{h}}, \quad \beta_{i i^{\prime}}=\sum_{j} \frac{V_{j i} \Gamma_{i^{\prime} j}}{K_{i j} \phi_{i}}, \\
\alpha_{i}=\sum_{j} \frac{V_{i j} R_{j}}{K_{i j} \phi_{i}}-D, \quad \beta_{i h}=\lambda_{h i} \beta_{h i}+\sum_{i^{\prime}} \Gamma_{h i^{\prime}} \beta_{i i^{\prime}}, \\
h=1, \ldots, H, \quad i, i^{\prime}=H+1, \ldots, I, \quad j=I+1, \ldots, J .
\end{gathered}
$$

A complete outcomes analysis is much beyond the purview of this paper, 
but two points are worth a mention in passing. Let the numbers of predators, prey and substrates at equilibrium be denoted by $N_{H}, N_{I}$ and $N_{J}$, respectively. (1) The number of supportable prey is not more than the sum of the number of resources and the number of supportable predators, i.e., $N_{I} \leqslant$ $N_{J}+N_{H}$. (2) The number of supportable predators is no greater than the number of supportable prey, i.e., $N_{H} \leqslant N_{I}$. Thus, $N_{H} \leqslant N_{I} \leqslant N_{J}+N_{H}$ or $0 \leqslant$ $N_{I}-N_{H} \leqslant N_{J}$. The first contention is easily established, since the matrix $\mathbf{B}$ may be partitioned as before, i.e., $\mathbf{B}=\mathbf{B}_{H}+\sum_{j} \mathbf{B}_{j}^{*}$. The matrix $\mathbf{B}_{H}$ may be written as

$$
\begin{aligned}
& \mathbf{B}_{H}=\left[\begin{array}{c:c}
\mathbf{0} & \mathbf{B}_{h i} \\
\hdashline \mathbf{B}_{i h} & \mathbf{0}
\end{array}\right] \begin{array}{l}
\left(N_{H}\right) \\
\left(N_{I}\right)
\end{array}, \\
& \left(N_{H}\right) \quad\left(N_{I}\right)
\end{aligned}
$$

where $\mathbf{B}_{h i}=\left\{\beta_{h i}\right\}$ and $\mathbf{B}_{i h}=\left\{\lambda_{h i} \beta_{h i}\right\}$, and $\mathbf{B}_{H}$ is thus of rank $R=\min \left(2 N_{H}\right.$, $\left.N_{H}+N_{I}\right)$. The matrix $\mathrm{B}_{j}^{*}$ takes the form

$$
\begin{gathered}
\mathbf{B}_{j}^{*}=\left[\begin{array}{c:c}
\mathbf{0} & \mathbf{0} \\
\hdashline \tilde{\mathbf{B}}_{j} & \mathbf{B}_{j}
\end{array}\right]_{\left(N_{I}\right)}^{\left(N_{H}\right)}, \\
\left(N_{H}\right) \quad\left(N_{I}\right)
\end{gathered}
$$

where $\mathbf{B}_{j}=\left\{V_{i j} \Gamma_{i^{\prime} j} / K_{i j} \phi_{i}\right\}$ and $\widetilde{\mathbf{B}}_{j}=\left\{\sum_{i^{\prime}}\left(\Gamma_{h i^{\prime}} V_{i j} \Gamma_{i^{\prime} j} / K_{i j} \phi_{i}\right)\right\}$. The matrix $\mathbf{B}_{j}^{*}$ is clearly of the same rank as $\mathbf{B}_{j}$, i.e., $N_{J}$. Thus, the rank of $\mathbf{B}$ is $\left(N_{j}+2 N_{H}\right)$, since $N_{H} \leqslant N_{I}$ (see below). The total number of supportable genotypes is no more than $\left(N_{J}+2 N_{H}\right)$, and since $N_{H}$ of these are predators, it follows that $N_{I} \leqslant N_{J}+N_{H}$. The proof that $N_{H} \leqslant N_{I}$ is trivial. The matrix B may now be written as

$$
\mathbf{B}=\left[\begin{array}{c:c}
0 & \mathbf{B}_{h l} \\
\hdashline \mathbf{B}_{i h}+\sum_{j} \widetilde{\mathbf{B}}_{j} & \sum_{j} \mathbf{B}_{j}
\end{array}\right]{ }_{\left(N_{I}\right)}^{\left(N_{H}\right)} .
$$

The determinant is the sum of many terms, each of which is the product of exactly $\left(N_{H}+N_{I}\right)$ elements. For a given term, each row and column must be represented once and only once. If $N_{H}>N_{l}$, at least one of the zero elements of the $\left(N_{H} \times N_{H}\right) 0$-matrix must be included in each term of the determinant, and the determinant must be zero. We therefore must have $N_{H} \leqslant N_{I}$. Full rank of the matrix $B$ is necessary for existence of a complete set of components. It is not sufficient. The stability conditions are rather more elaborate, and can be obtained in any particular case by recourse to the procedures illustrated in the Appendix. 


\section{Food Chains and Food Webs}

The purpose of this section is to indicate very briefly the extensions necessary to construct multi-layered food chains and fod webs. An exhaustive treatment is unnecessary, because a few examples will illustrate the points of interest.

Multi-layered predation. Consider a set of "super-predators" $\left(G_{f}: f=\right.$ $1, \ldots, F)$, which feed on a set of predators $\left(G_{h}: i=H+1, \ldots, I\right)$, which feed on a set of substrates $\left(S_{j}: j=I+1, \ldots, J\right)$. A schematic for one super-predator, one predator, one prey and one substrate is presented in Fig. 3a. Following the strategy utilized above, the system may be described by

$$
\begin{gathered}
\dot{M}_{f}=\sum_{h}\left[\frac{V_{f h} M_{h}}{K_{f h} \phi_{f}}-\gamma_{f h} D\right] M_{f}=\sum_{h} \dot{M}_{f h}, \\
\dot{M}_{h}=\sum_{i}\left[\frac{V_{h i} M_{i}}{K_{h i} \phi_{h}}-\gamma_{h i}\left(D+\sum_{f} \frac{V_{f h} \lambda_{f h}}{K_{f h} \phi_{f}} M_{f}\right)\right] M_{h}=\sum_{i} \dot{M}_{h i}, \\
\dot{M}_{i}=\sum_{j}\left[\frac{V_{i j} C_{j}}{K_{i j} \phi_{i}}-\gamma_{i j}\left(D+\sum_{h} \frac{V_{h i} \lambda_{h i}}{K_{h i} \phi_{h}} M_{h}\right)\right] M_{i}=\sum_{j} \dot{M}_{i j}, \\
\dot{C}_{j}=\left(R_{j}-C_{j}\right) D-\sum_{i} \frac{V_{i j} \lambda_{i j}}{K_{i j} \phi_{i}} M_{i}, \\
\left.\dot{C}_{j}+\sum_{i} \lambda_{i j}\left(\dot{M}_{i j}+\gamma_{i j} \sum_{h} \lambda_{h i}\left\{\dot{M}_{h i}+\gamma_{h i} \sum_{f} \lambda_{f h} \dot{M}_{f h}\right\}\right)\right] \\
=\left[R_{j}-C_{j}-\sum_{i} \Gamma_{i j}\left(M_{i}+\sum_{h} \Gamma_{h i}\left\{M_{h}+\sum_{f} \Gamma_{f h} M_{f}\right\}\right)\right] D \approx 0 .
\end{gathered}
$$

Here $\phi_{f}=\left[1+\sum_{h} K_{f h}^{-1} M_{h}\right], \phi_{h}=\left[1+\sum_{i} K_{h i}^{-1} M_{i}\right]$ and $\phi_{i}=\left[1+\sum_{j} K_{i j}^{-1} C_{j}\right]$. We are led to the three-layered system

$$
\begin{aligned}
& \dot{M}_{f}=\left[-\alpha_{f}+\sum_{h} \beta_{f h} M_{h}\right] M_{f}, \\
& \dot{M}_{h}=\left[-\alpha_{h}-\sum_{f} \beta_{h f} M_{f}+\sum_{i} \beta_{h i} M_{i}\right] M_{h}, \\
& \dot{M}_{i}=\left[\alpha_{i}-\sum_{f} \beta_{i f} M_{f}-\sum_{h} \beta_{i h} M_{h}-\sum_{i^{\prime}} \beta_{i i^{\prime}} M_{i^{\prime}}\right] M_{i},
\end{aligned}
$$


with the $\alpha$ - and $\beta$-coefficients given by

$$
\begin{gathered}
\alpha_{f}=D=\alpha_{h}, \quad \alpha_{i}=\sum_{j} \frac{V_{i j} R_{j}}{K_{i j} \phi_{i}}-D, \\
\beta_{f h}=\frac{V_{f h}}{K_{f h} \phi_{f}}, \quad \beta_{h f}=\frac{V_{f h} \lambda_{f h}}{K_{f h} \phi_{f}}, \quad \beta_{h t}=\frac{V_{h i}}{K_{h i} \phi_{h}}, \\
\beta_{i f}=\sum_{h} \sum_{i} \Gamma_{f h} \Gamma_{h i^{\prime}} \beta_{i i^{\prime}}, \quad \beta_{i h}=\lambda_{h i} \beta_{h i}+\sum_{i^{\prime}} \Gamma_{h i^{\prime}} \beta_{i i^{\prime}}, \\
\beta_{i i^{\prime}}=\sum_{j} \frac{V_{i j} \lambda_{i^{\prime} j}}{K_{i j} \phi_{i}} .
\end{gathered}
$$

Denote the equilibrium numbers of super-predators, predators, prey and substrates by $N_{F}, N_{H}, N_{I}$ and $N_{J}$, respectively. Using B-matrix partitions and singularity arguments, it can be shown that the existence of an equilibrium with all components present requires

$$
\begin{array}{lll}
\left(N_{F} \leqslant N_{H} \leqslant N_{I}+N_{F}\right) & \text { or } & \left(0 \leqslant N_{H}-N_{F} \leqslant N_{I}\right), \\
\left(N_{H} \leqslant N_{I} \leqslant N_{J}+N_{H}\right) & \text { or } & \left(0 \leqslant N_{I}-N_{H} \leqslant N_{J}\right) .
\end{array}
$$

The extension to four or more trophic layers is entirely obvious, as is the multi-layered extension of (25). One other point worth noting is that stable limit cycles are not inherent in any of these models, because of the self- and cross-damping terms within the bottommost (prey) layer. These, in turn, depend on the explicit relation between the prey and the renewable resources.

Facultative predation. The model is very easily modified to allow trophic level crossing by one or more organisms. One may construct complicated food webs in this fashion. I shall describe here only a pair of simple cases, simply to indicate the pattern of the extension. Consider first the one predator: one prey case described earlier, but suppose that the predator $\left(G_{1}\right)$ may bypass the prey $\left(G_{2}\right)$ to use the substrates directly. A schematic for the single substrate case of "trophic bypass" is presented in Fig. 3b. The system is now described by

$$
\begin{gathered}
\dot{M}_{1}=\left[\frac{V_{12} M_{2}}{K_{12} \phi_{1}}-\gamma_{12} D\right] M_{1}+\sum_{j}\left[\frac{V_{1 j} C_{j}}{K_{1 j} \phi_{1}}-\gamma_{1 j} D\right] M_{1}=\dot{M}_{12}+\sum_{j} \dot{M}_{1 j}, \\
\dot{M}_{2}=\sum_{j}\left[\frac{V_{2 j} C_{j}}{K_{2 j} C_{j}}-\gamma_{2 j}\left(D+\frac{V_{12} \lambda_{12}}{K_{12} \phi_{1}} M_{1}\right)\right] M_{2}=\sum_{j} \dot{M}_{2 j} \\
\dot{C}_{j}=\left(R_{j}-C_{j}\right) D-\frac{V_{1 j} \lambda_{1 j}}{K_{1 j} \phi_{1}} M_{1}-\frac{V_{2 j} \lambda_{2 j}}{K_{2 j} \phi_{2}} M_{2} \\
{\left[\dot{C}_{j}+\lambda_{2 j}\left(\dot{M}_{2 j}+\gamma_{2 j} \lambda_{12} \dot{M}_{12}\right)+\lambda_{1 j} \dot{M}_{1 j}\right]} \\
=\left[R_{j}-C_{j}-\Gamma_{2 j} M_{2}-\left(\Gamma_{12} \Gamma_{2 j}+\Gamma_{1 j}\right) M_{1}\right] D \approx 0 .
\end{gathered}
$$



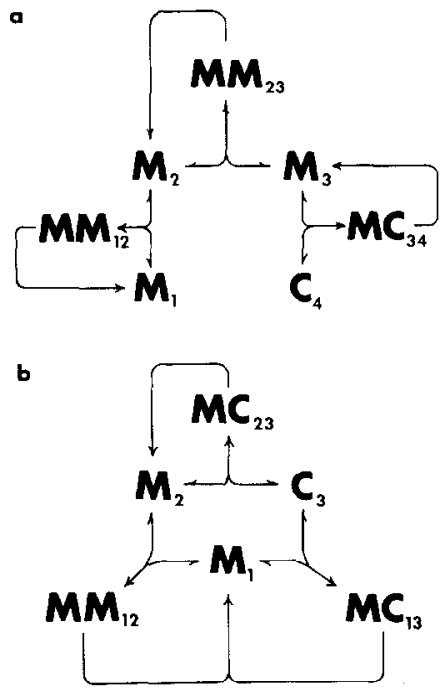

FIG. 3. Trophic layer models: (a) Superpredator model, with $M_{1}, M_{2}$ and $M_{3}=$ masses of the superpredator, predator and prey, respectively, and $C_{4}=$ concentration of the limiting substrate; (b) Predation model with trophic bypass, with $M_{1}$ and $M_{2}=$ masses of the facultative predator and prey, respectively, and $C_{3}=$ concentration of a limiting substrate.

Note here that $\phi_{1}=\left[1+K_{12}^{-1} M_{2}+\sum_{j} K_{1 j}^{-1} C_{j}\right]$. One is led to the apparent Lotka-Volterra competition model

$$
\begin{aligned}
& M_{1}=\left[\alpha_{1}-\beta_{11} M_{1}-\beta_{12} M_{2}\right] M_{1}, \\
& M_{2}=\left[\alpha_{2}-\beta_{21} M_{1}-\beta_{22} M_{2}\right] M_{2},
\end{aligned}
$$

where the $\alpha$ - and $\beta$-coefficients are given by

$$
\begin{gathered}
\alpha_{1}=\sum_{j} \frac{V_{1 j} R_{j}}{K_{1 j} \phi_{1}}-D, \quad \sum_{j} \frac{V_{2 j} R_{j}}{K_{2 j} \phi_{2}}-D=\alpha_{2}, \\
\beta_{11}=\sum_{j} \frac{V_{1 j}\left(\Gamma_{1 j}+\Gamma_{12} \Gamma_{2 j}\right)}{K_{1 j} \phi_{1}}, \quad \sum_{j} \frac{V_{2 j} \Gamma_{2 j}}{K_{2 j} \phi_{2}}=\beta_{22}, \\
\beta_{12}=\frac{V_{1 j} \Gamma_{2 j}}{K_{1 j} \phi_{1}}-\frac{V_{12}}{K_{12} \phi_{1}}, \quad \frac{V_{12} \lambda_{12}}{K_{12} \phi_{1}}+\sum_{j} \frac{V_{2 j}\left(\Gamma_{1 j}+\Gamma_{12} \Gamma_{2 j}\right)}{K_{2 j} \phi_{2}}=\beta_{21} .
\end{gathered}
$$

Because $\beta_{12}$ may be either positive or negative, we really have a mixed predation-competition model. The outcomes analysis is more complicated than that of either the pure competition model (Smouse, 1980) or the simple predation model (see above). Suffice it to say that the predator may persist even in the absence of the prey, provided $\alpha_{1}>0$ with $\hat{M}_{2}=0$. 
Now, consider a two predator, one prey case. Recall that for pure predation models, we require $N_{H} \leqslant N_{I}$ for the existence of all components simultaneously. Suppose, however, that one of the predators $\left(G_{2}\right)$ may facultatively bypass the prey $\left(G_{3}\right)$, and may use the substrate $\left(C_{j}\right)$ layer. The altered model is given by

$$
\begin{gathered}
\dot{M}_{1}=\left[\frac{V_{13} M_{3}}{K_{13} \phi_{1}}-D\right] M_{1}, \\
\dot{M}_{2}=\left[\frac{V_{23} M_{3}}{K_{23} \phi_{2}}-\gamma_{23} D\right] M_{2}+\sum_{j}\left[\frac{V_{2 j} C_{j}}{K_{2 j} \phi_{2}}-\gamma_{2 j} D\right] M_{2} \\
=\dot{M}_{23}+\sum_{j} \dot{M}_{2 j}, \\
\dot{M}_{3}=\sum_{j}\left[\frac{V_{3 j} C_{3}}{K_{3 j} \phi_{3}}-\gamma_{3 j}\left(D+\frac{V_{23} \lambda_{23}}{K_{23} \phi_{2}} M_{2}\right)\right] M_{3}, \\
\dot{C}_{j}=\left(R_{j}-C_{j}\right) D-\frac{V_{2 j} \lambda_{2 j}}{K_{23} \phi_{2}} M_{2}-\frac{V_{3 j} \lambda_{3 j}}{K_{3 j} \phi_{3}} M_{3}, \\
{\left[\dot{C}_{j}+\lambda_{3 j}\left(\dot{M}_{3 j}+\gamma_{3 j} \lambda_{13} \dot{M}_{1}+\gamma_{3 j} \lambda_{23} \dot{M}_{23}\right)+\lambda_{2 j} \dot{M}_{2 j}\right]} \\
=\left[R_{j}-C_{j}-\Gamma_{3 j} M_{3}-\lambda_{13} \Gamma_{3 j} M_{1}-\left(\Gamma_{23} \Gamma_{3 j}+\Gamma_{2 j}\right) M_{2}\right] D \approx 0 .
\end{gathered}
$$

Obviously $\phi_{2}=\left[1+K_{23}^{-1} M_{3}+\sum_{j} K_{2 j}^{-1} C_{j}\right]$. One is led to the model

$$
\begin{aligned}
& \dot{M}_{1}=\left[-\alpha_{1}+\beta_{13} M_{3}\right] M_{1}, \\
& M_{i}=\left[\alpha_{i}-\beta_{i 1} M_{1}-\sum_{i^{\prime}} \beta_{i i^{\prime}} M_{i^{\prime}}\right] M_{i}, \quad i, i^{\prime}=2,3 .
\end{aligned}
$$

where the $\alpha$ - and $\beta$-coefficients are given by

$$
\begin{gathered}
\alpha_{1}=D, \quad \alpha_{i}=\sum_{j} \frac{V_{i j} R_{j}}{K_{1 j} \phi_{i}}-D, \quad \beta_{13}=\frac{V_{13}}{K_{13} \phi_{1}}, \\
\beta_{21}=\Gamma_{13} \sum_{j} \frac{V_{2 j} \Gamma_{3 j}}{K_{2 j} \phi_{2}}, \quad \beta_{31}=\Gamma_{13} \beta_{33}, \quad \beta_{33}=\sum_{j} \frac{V_{3 j} \Gamma_{33}}{K_{3 j} \phi_{3}}, \\
\beta_{22}=\sum_{j} \frac{V_{2 j}\left(\Gamma_{2 j}+\Gamma_{23} \Gamma_{3 j}\right)}{K_{2 j} \phi_{2}}, \quad \beta_{23}=\sum_{j} \frac{V_{2 j} \Gamma_{3 j}}{K_{2 j} \phi_{2}}-\frac{V_{23}}{K_{23} \phi_{2}} \\
\beta_{32}=\frac{\lambda_{23} V_{23}}{K_{23} \phi_{2}}+\sum_{j} \frac{V_{3 j}\left(\Gamma_{2 j}+\Gamma_{23} \Gamma_{3 j}\right)}{K_{3 j} \phi_{3}} .
\end{gathered}
$$

Because $\beta_{23}$ may be either positive or negative, the analysis of steady-states for this hybrid model is rather complicated. Although $G_{1}$ may not persist in the absence of $G_{3}, G_{2}$ may do so if $\alpha_{2}>0$ with $\hat{M}_{3}=0$. Morever, both 
predators may persist with a single prey, and for this case $N_{H}>N_{I}$. It is easily seen how this sort to trophic "leap-frogging" may be generalized to multi-layered systems. At the cost of rapidly increasing complexity, one may model a great variety of trophic structures and food webs.

\section{Discussion}

The relations (25) relating supportable numers of genotypes (species) in different trophic layers have some interesting implications for the evolution of ecosystems in the chemostat (or for that matter in nature). Imagine that one begins with the array $\left(N_{F}=0: N_{H}=0: N_{l}=1: N_{J}=1\right)$. Since any organism is capable of mutating, one might well expect the time sequence $(0: 0: 1: 1) \rightarrow(0: 1: 1: 1) \rightarrow(0: 1: 2: 1) \rightarrow(0: 2: 2: 1) \rightarrow(0: 2: 3: 1) \rightarrow(1: 2: 3: 1) \rightarrow$ $(1: 3: 3: 1) \rightarrow(2: 3: 3: 1) \rightarrow(2: 4: 3: 1)$, and so on. When one considers that trophic layer crossing is also likely to develop, it would seem that the trophic pyramid could (in principle) build on its own complexity. That a certain amount of this sort of thing can actually occur in the chemostat is attested by the bacteria:phage experiments of Horne (1971) and Chao et al. (1977). The phenomenon is real. Moreover, its theoretical justification is not a special feature of the models considered here. Levin et al. (1977) come to the same conclusion, using a rather more general (and less specific) model than that employed here; the result itself would appear to be robust to details of the model. Why is it, then, that one normally finds $\left(N_{F}<N_{H}<N_{I}<N_{J}\right)$ ? To begin with, the statement (25) is rather weak; this claim rests on the nonsingularity of the B-matrix. Full rank for B is necessary but not sufficient to guarantee either the existence or the stability of a particular mixed equilibrium. The additional specifications required to guarantee existence and stability of complex steady states become ever more restrictive on the parameter space as the number of components is increased. Moreover, local stability for a particular community (all that is being assessed with the $\mathbf{B}$ matrix) does not imply global stability for that same equilibrium (cf. Richardson and Smouse, 1975; Goh, 1977). It is not uncommon for such models to admit of several locally stable equilibria, most of which will involve only small numbers of upper trophic level components. One might conjecture that the domains of local attraction for such side-solutions would generally be larger than those of more elaborate (and more exquisitely balanced) equilibria. In any case, communities must generally evolve from the bottom-up, and most communities may never escape the domains of attraction for the simpler side-solutions.

Although not an inherent feature of the models proposed, it is also clear that the energetic conversion efficiency between adjacent layers is low, i.e., the $\lambda$-values are quite large. For the upper trophic layers, the available 
energy is minimal, and population sizes will be correspondingly small. Under such circumstances, the stability of the whole assemblage is vulnerable to stochastic perturbation (cf. Bartlett, 1957; Leslie and Gower, 1958; Goh, 1976; Chesson, 1978). The periodicities introduced by lag phenomena are real features of layered systems (eg. Cunningham, 1954; Wangersky and Cunningham, 1957; Luckinbill, 1973; May and Oster, 1976; Wangersky, 1978), and exacerbate the stochastic instabilities. Considering all of these features, it is not surprising that most trophic pyramids are relatively flat. One should expect not only that $\left(N_{F}<N_{H}<N_{I}<N_{J}\right)$ but that the inequalities will be large under most circumstances.

Finally, a few words are in order about the utility of translating the more information-rich chemostat models into the quadratic Lotka-Volterra analogues used here. All too often, the parameters of these latter models are simply invoked. It is useful (indeed chastening) to obtain an appreciation of just how much information these $\alpha$ - and $\beta$-"parameters" actually subsume. This comment notwithstanding, it is useful to make the translation. A comparison of models (22) or (26), for example, with their analogues (23) and (27), should convince the reader that some condensation of information is desirable. The numbers of parameters in the full chemostat models are simply overwhelming. Except in fairly simple cases, the task of estimating all of these parameters is prohibitive, even with so powerful an experimental tool as the chemostat. For simple predation (or host-parasite) systems, chemostats and chemostat theory of the general sort propounded here have already proven their utility (cf. Horne, 1971; Tsuchiya et al., 1972; Luckinbill, 1973; Drake and Tsuchiya, 1976, 1977; Chao et al., 1977; Levin et al., 1977). It seems probable that this sort of work may be profitably (but modestly) extended to multiple substrates and to an additional trophic layer or two. For really elaborate communities, however, the models will remain purely theoretical tools. It seems clear that for a general treatment of complicated systems, we may have to turn to the sort of generalized network analysis proposed hy Levins (1974) or to linearized systems theory. That being the case, we must be prepared to sacrifice exquisite detail by means of some "judicious condensation" of information. The translation into classic Lotka-Volterra form is just such a condensation. The $\alpha$ - and $\beta$-parameters are a convenient and useful visualization, but should never be taken too literally.

\section{SUMMARY}

Models are presented for the joint dynamics of predators and prey, maintained in continuous flow chemostat culture. The predators are visualized as subsisting on one or more prey organisms, which in turn are visualized as 
subsisting on one or more substrate resources supplied by the investigator. The dynamic equations are translated into an analogous Lotka-Volterra predation model, and the criteria for the existence and stability of various equilibria are indicated. Denoting the number of different predator organisms as $N_{H}$, the number of different prey organisms by $N_{I}$ and the number of different substrates as $N_{J}$, it is shown that the joint coexistence of all components requires $0 \leqslant N_{t}-N_{H} \leqslant N_{J}$. The model is extended to more complex situations by including additional trophic layers and by allowing trophic layer "leap-frogging." The model may always be translated into an approximately quadratic differential equation of the Lotka-Volterra type. The $\alpha$ - and $\beta$-coefficients of these latter are really variables, and become quite complex for some of the multi-layered models.

\section{APPENDIX}

The purpose of this appendix is to illustrate the analysis of stability criteria. I shall briefly indicate the strategy for the one predator, one prey, one substrate and two predator; one prey, one substrate cases below, illustrations which should indicate the general pattern of the other solutions.

One predator, one prey, one substrate. The text model (5), describing the dynamics of $M_{1}, M_{2}$, and $C_{3}$, yields the Jacobian

$\mathbf{J}_{M C}=\left[\begin{array}{ccc}\left(\mu_{12}-D\right) & M_{1} \frac{\partial \mu_{12}}{\partial M_{2}} & 0 \\ -\lambda_{12} \mu_{12} & \left(\mu_{23}-D\right)-\lambda_{12} M_{1} \frac{\partial \mu_{12}}{\partial M_{2}} & M_{2} \frac{\partial \mu_{23}}{\partial C_{3}} \\ 0 & -\lambda_{23} \mu_{23} & -D-\lambda_{23} M_{2} \frac{\partial \mu_{23}}{\partial C_{3}}\end{array}\right]$

It is most convenient to extract the characteristic roots of the equivalent Jacobian $\mathbf{J}_{X}=\mathbf{P J}_{M C} \mathbf{P}^{-1}$, where the transformation matrix $\mathbf{P}$ takes the form

$$
\mathbf{P}=\left[\begin{array}{ccc}
1 & 0 & 0 \\
0 & 1 & 0 \\
\lambda_{12} \lambda_{23} & \lambda_{23} & 1
\end{array}\right],
$$

and the matrix $\mathbf{J}_{X}$ takes the form 


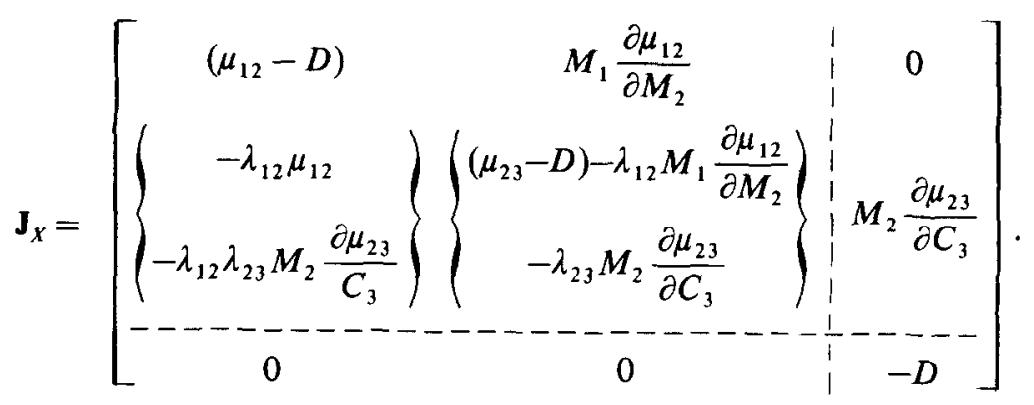

For Case I $\left(\hat{M}_{1}=0=\hat{M}_{2}, \hat{C}_{3}=R_{3}\right)$, the eigenequation takes the form

$$
(-D-w)\left(\mu_{12}-D-w\right)\left(\mu_{23}-D-w\right)=0
$$

and local stability requires $\mu_{12}\langle D\rangle \mu_{23}$ with $\hat{M}_{2}=0$. Now, $\mu_{12}=0$ if $\hat{M}_{2}=0$, and $D>\mu_{23}$ implies that

$$
\frac{\alpha_{2}}{\beta_{22}}=\frac{\left(\theta_{23} R_{3}-D\right)}{\theta_{23} \lambda_{23}}<0
$$

as claimed in the text. Case II $\left(\hat{M}_{1}=0<\hat{M}_{2}, \hat{C}_{3}<R_{3}\right)$ reduces the eigenequation to the form

$$
(-D-w)\left(\mu_{12}-D-w\right)\left(\mu_{23}-D-\lambda_{23} M_{2} \frac{\partial \mu_{23}}{\partial C_{3}}-w\right)=0 .
$$

For this steady state, $\left(\mu_{23}-D\right)=0$, so the third term yields a negative root whenever $\hat{M}_{2}>0$, because $\partial \mu_{23} / \partial C_{3}>0$. If $\left(\mu_{12}-D\right)<0$, then $\hat{M}_{2}<D \theta_{12}^{-1}=$ $\alpha_{1} / \beta_{12} ;$ if $\hat{M}_{2}>0$, then $\theta_{23} R_{3}>D$ and

$$
\frac{\alpha_{2}}{\beta_{22}}=\frac{\theta_{23} R_{3}-D}{\theta_{23} \lambda_{23}}<D \theta_{12}^{-1}=\frac{\alpha_{1}}{\beta_{12}},
$$

which is the required Liapanov stability condition indicated in the text. For Case III $\left(\hat{M}_{1}>0<\hat{M}_{2}, \hat{C}_{3}<R_{3}\right)$, the steady state is stable if $D>0$ (which it is) and if both roots $(w)$ are negative from

$$
w=-b \pm \sqrt{b^{2}-4 a},
$$

where

$$
\begin{aligned}
& a=\left(\frac{\lambda_{12} V_{12} M_{1}}{K_{12} \phi_{1}^{2}}\right) \times\left(D+\frac{\lambda_{23} V_{23} M_{2}}{K_{23} \phi_{2}^{2}}\right), \\
& b=\left(\frac{\lambda_{12} V_{12} M_{1}}{K_{12} \phi_{1}^{2}}-\mu_{23}\right)+\left(D+\frac{\lambda_{23} V_{23} M_{2}}{K_{23} \phi_{2}^{2}}\right) .
\end{aligned}
$$


A bit of manipulation shows that both $(a)$ and $(b)$ are positive (and the roots (w) are negative) if the inequality in (A7) is reversed. This is the stability condition invoked in the text.

Two predators, one prey, one substrate. Consider the two-predator, oneprey, one substrate version of text model (16), describing the dynamics of $M_{1}, M_{2}, M_{3}$ and $C_{4}$. The Jacobian of this model is of the form

$$
\mathbf{J}_{M C}=\left[\begin{array}{cccc}
\left(\mu_{13}-D\right) & 0 & M_{1} \frac{\partial \mu_{13}}{\partial M_{3}} & 0 \\
0 & \left(\mu_{23}-D\right) & M_{2} \frac{\partial \mu_{23}}{\partial M_{3}} & 0 \\
-\lambda_{13} \mu_{13} & -\lambda_{23} \mu_{23} & (a) & M_{3} \frac{\partial \mu_{34}}{\partial C_{4}} \\
0 & 0 & -\lambda_{34} \mu_{34} & (d)
\end{array}\right],
$$

where

$$
\begin{aligned}
& (a)=\left(\mu_{34}-D\right)-\lambda_{13} \frac{\partial \mu_{13}}{\partial M_{3}}-\lambda_{23} \frac{\partial \mu_{23}}{\partial M_{3}}, \\
& (d)=-D-\lambda_{34} M_{3} \frac{\partial \mu_{34}}{\partial C_{4}} .
\end{aligned}
$$

Using a transform matrix $\mathbf{P}$, one may evaluate the eigenvalues of the matrix $\mathbf{J}_{X}=\mathbf{P J}_{M C} \mathbf{P}^{-1}$ instead. The matrix $\mathbf{P}$ takes the form

$$
\mathbf{J}_{X}=\left[\begin{array}{cccc}
1 & 0 & 0 & 0 \\
0 & 1 & 0 & 0 \\
\lambda_{13} & \lambda_{23} & 1 & 0 \\
\lambda_{13} \lambda_{34} & \lambda_{23} \lambda_{34} & \lambda_{34} & 1
\end{array}\right] .
$$

The stability conditions for all cases are of the usual Lotka-Volterra sort to be derived from text model (17). I shall comment here only on the steady state $\left(\hat{M}_{1}, \hat{M}_{2}, \hat{M}_{3}>0, C_{4}<R_{4}\right)$. This particular steady state reduces (A10) to the form

$$
\mathbf{J}_{M C}=\left[\begin{array}{cccc}
0 & 0 & + & 0 \\
0 & 0 & + & 0 \\
- & - & - & + \\
0 & 0 & - & -
\end{array}\right] .
$$

Every element of det $\mathbf{J}_{M C}$ is multiplied by one or more of the zeros in the first two rows of (A13); $J_{M C}$ is thus singular, so that the usual Liapanov 
treatment does not apply. Further investigation indicates that there can be no $\left(\hat{M}_{1}, \hat{M}_{2}, \hat{M}_{3}>0, \hat{C}_{4}<R_{4}\right)$ solution to text model (16). This situation is as indicated in the text, and follows from the B-matrix singularity arguments. It develops that $\mathbf{B}$ is singular iff $\mathbf{J}_{M C}$ is singular. No more predators may persist than prey.

Other models may be investigated in analogous fashion. As the number of components to the system increases, the translation to Lotka-Volterra stability conditions becomes more tedious and less useful, but should always be possible.

\section{ACKNOWLEDGMENTS}

I would particularly like to thank Dr. Patricia Moll and Dr. Curtis Strobeck, whose myriad suggestions greatly improved the paper. The responsibility for the formulation presented as well as for any errors of omission or commission, remains my own.

\section{REFERENCES}

BARTLETT, M. S. 1957. On theoretical models for competitive and predatory biological systems, Biometrika 44, 27-42.

Canale, R. P. 1969. Predator-prey relationships in a model for the activated process, Biotech. Bioeng. 11, 887-907.

Canale, R. P. 1970. An analysis of models describing predator-prey interaction, Biotech. Bioeng. 12, 353-378.

Chao, L., Levin, B. R., ANd Stewart, F. M. 1977. A complex community in a simple habitat: an experimental study with bacteria and phage, Ecology 58, 369-378.

Chesson, P. 1978. Predator-prey theory and variability, Ann. Rev. Ecol. Syst. 9, 323-347.

Cunningham, W. J. 1954. A non-linear differential-difference equation of growth, Proc. Nat. Acad. Sci. USA 40, 708-713.

Drake, J. F., AND TsuchiYa, H. M. 1976. Predation on Escherichia coli by Colpoda steinii, Appl. Env. Micrubivl. 31, 870-874.

Drake, J. F., AND TsuchiYa H. M. 1977. Growth kinetics of Colpoda steinii on Escherichia coli, Appl. Env. Microbiol. 34, 18-22.

Gause, G. F. 1934. "The Struggle for Existence," Williams \& Wilkins, Baltimore.

GiLPIN, M. E. 1974. A Liapunov function for competition communities, J. Theor. Biol. 44, $35-48$.

GoH, B. S. 1976. Nonvulnerability of ecosystems in unpredictable environments, Theor. Pop. Biol. 10, 83-95.

GoH, B. S. 1977. Global stability in many-species systems, Amer. Natur. 111, 135-143.

HORNE, M. T. 1971. Coevolution of Escherichia coli and bacteriophages in chemostat culture, Science 168, 992-993.

Jost, J. L., Drake, J. F., Tsuchiya, H. M., And Fredrickson, A. G. 1973. Microbial food chains and food webs, J. Theor. Biol. 41, 461-484.

Leslie, P. H., AND Gower, J. C. 1958. The properties of a stochastic model for two competing species, Biometrika 45, 316-330. 
Levin, B. R., Stewart, F. M., and Chao, L. 1977. Resource limited growth, competition and predation: a model and experimental studies with bacteria and bacteriophage, Amer. Natur. 111, 3-24.

Levins, R. 1974. The qualitative analysis of partially specified systems, Ann. N. Y. Acad. Sci. 231, 123-138.

LotKA, A. 1925. "Elements of Physical Biology," Williams \& Wilkins, Baltimore.

LUCKINBILL, L. S. 1973. Coexistence in laboratory populations of Paramecium aurelia and its predator Didinium nasutum, Ecology 54, 1320-1327.

MAY, R. M. 1973. Limit cycles in predator-prey communities, Science 177, 900-902.

MAY, R. M., AND OsTER, G. F. 1976. Bifurcations and dynamic complexity in simple ecological models, Amer. Natur. 110, 573-579.

MonOD, J. 1949. The growth of bacterial cultures, Ann. Rev. Microbiol. 3, 371-394.

Richardson, R. H., and Smouse, P. E. 1975. Ecological specialization of Hawaiian Drosophila. II. The community matrix, ecological complementation, and phyletic species packing, Oecologia 22, 1-13.

Schoener, T. W. 1973. Population growth regulated by intraspecific competition for energy or time: Some simple representations, Theor. Pop. Biol. 4, 56-85.

SChoener, T. W. 1974. Competition and the form of habitat shift, Theor. Pop. Biol. 6, $265-307$.

SMouse, P. E. 1980. Mathematical models for continuous culture growth dynamics of mixed populations subsisting on a heterogeneous resource base. I. Simple competition, Theor. Pop. Biol. 17, 16-36.

StrobeCK, C. 1973. $N$ species competition, Ecology 54, 650-654.

Tsuchiya, H. M., Drake, J. F., Jost, J. L., and Frederickson, A. G. 1972. Predator-prey interactions of Dictyostelium discoides and Escherichia coli in continuous cultures, J. Bact. $110,1147-1153$.

Volterra, V. 1926. "La Lutte Pour La Vie," Gauthier-Villars, Paris.

Waldon, M. G. 1975. Competition models, Amer. Natur. 109, 487-489.

WANGerSKy, P. J. 1978. Lotka-Volterra population models, Ann. Rev. Ecol. Syst. 9, 189-218.

WANGERSKY, P. J., AND CunNinghaM, W. J. 1957. Time lag in prey-predator population models, Ecology 38, 136-139. 